\title{
The Effect of Helfer Skin Tap Technique and ShotBlocker Application on Pain in Deltoid Muscle Injection
}

\author{
Tuba Karabey ${ }^{1}$, Serife Karagozoglu ${ }^{\circledR}$ \\ ${ }^{1}$ Gaziosmanpasa University Faculty of Health Science, Division of Nursing, Department of Fundamentals of Nursing, Tokat, Turkey. \\ ${ }^{2}$ Cumhuriyet University Faculty of Health Science, Division of Nursing, Department of Fundamentals of Nursing, Sivas, Turkey.
}

Correspondence Author: Tuba Karabey

E-mail: tubakarabey @hotmail.com

Received: $15.01 .2021 \quad$ Accepted: 02.11.2021

\begin{abstract}
Objective: This study was performed experimentally and single-blind randomized controlled trial to compare the effects of Helfer Skin Tap technique and Shot Blocker application on pain in the intramuscular injectıon application to the deltoid muscle.

Methods: The study was designed as a prospective, single-blind randomized controlled trial. The research was conducted in accordance with the Consolidated Standards of Reporting Trials (CONSORT) Checklist guide. The study was conducted in a family health center in Sivas Province between 05.10.2020-30.12.2020. Individuals who received Hepatitis B vaccine injection were included in the sample of the study. All intramuscular injections were made by the same nurse, and the approaches to reducing pain were made by the researcher. A total of 120 patients including the Helfer Skin Tap Technique $(n=40)$, the standard application $(n=40)$ and the ShotBlocker technique $(n=40)$ were included. Mann Whitney $\mathrm{U}$ and Kruskal Wallis tests were used to evaluate the data.

Results: In our study, 75\% ( $n=30$ ) of the Helfer Skin Tap group, $82.50 \%(n=33)$ of the standard administration group and $75 \%(n=30)$ of the ShotBlocker group reported that they had no fear of injection. When the distribution of post-injection pain scores of the individuals according to different injection methods was examined, the difference between the average pain scores of the different methods used to provide pain control was found to be significant and statistically significant $(p=0.001)$.

Conclusion: In line with the findings of our study, it was concluded that the use of ShotBlocker in intramuscular injection application of individuals was more effective in reducing pain due to injection compared to Helfer Skin Tap and standard application, and the Helfer Skin Tap technique was more successful in pain control than standard practice. All health professionals', especially nurses', awareness of the effectiveness of different methods in reducing pain during IM injection should be raised. To ensure pain control during IM injection, Shotblocker and Helfer Skın Tap Technıque should be preferred since they are easy to apply and cost effective,
\end{abstract}

Keywords: Intramuscular Injection, Pain Control, Helfer Skin Tap, Shot Blocker

\section{INTRODUCTION}

Intramuscular (IM) injection administration is an important part of parenteral drug administration and is a common nursing function that is frequently used in clinical practice $(1,2)$. Although considered as a simple intervention, IM injections cause very serious complications if they are not done with appropriate methods (2-4). In the studies conducted, it was determined that IM injections were among the most painful applications among invasive procedures performed in hospitals $(5,6)$. It has been reported that the pain that develops due to IM injection is due to the mechanical trauma caused by the needle insertion and the sudden pressure created when the drug is administered into the muscle (7). Because the injection area is small among the IM injection sites, the most painful area is the deltoid area (8). In this context, it is extremely important to use pain control approaches in IM injection applied to this area.
In the literature, it is emphasized that IM injection, which is applied based on guidelines and scientific knowledge, may experience less pain and prevent injection-related complications $(5,6,9)$. In this context, when the literature was examined, it was seen that different methods, techniques and approaches such as cold application during IM injection, internal rotation of the extremities, use of ShotBlocker, Helfer Skin Tap technique, acupuncture application, relaxation exercises and the $Z$ technique reduce injection pain. $(7,10,11)$ Helfer Skin Tap technique, one of these techniques, was developed by nurse Joanne Kieffer Helfer to reduce pain associated with IM injection (12). This technique is based on providing pain control by providing mechanical stimulation with rhythmic touching to the injection area, and it is an easyto-apply and time-consuming pain relief approach (13). IM injection applied to the dorsogluteal region using the Helfer 
Skin Tap technique has also been stated that this technique is an effective approach to relieve injection pain $(11,14)$.

Another method used to reduce injection pain is ShotBlocker application. ShotBlocker is a plastic tool that prevents the perception of pain in intramuscular injection and its transmission to the central nervous system by temporarily blocking the peripheral nerve endings. ShotBlocker is a small, flat, non-invasive, horseshoe (U-shaped) plastic tool that can be used for all age groups to reduce the pain associated with intramuscular injection, it can be used quickly and easily, inexpensive, does not require prior material preparation, It has properties used by keeping it on its surface (15).The proposed mechanism of action of ShotBlocker is that the pressure exerted on the skin by the protrusions in this tool stimulates smaller diameter and faster nerve endings $(15,16)$. This stimulation reduce spain by temporarily blocking the slower pain signals during injection, closing the gates to the central nervous system. Most of the published studies examining the effect of ShotBlocker in reducing intramuscular injection pain have been conducted with children $(16,17)$ and its use in adults has been very limited $(18,19)$. Nurses are responsible for preventing injection pain or relieving the patient with the techniques they use in drug administration. Pain due to intramuscular injection, which has physical and emotional effects, should be reduced in order to maintain patient-nurse relations, patient care quality and patient satisfaction $(16,18,19)$.

When the studies using Helfer Skin Tap and ShotBlocker techniques were examined, no study was found that evaluated the effectiveness of these two techniques in the application of deltoid muscle injection together. It is important to use evidence-based approaches in order to highlight the concept of quality in the delivery of nursing care. In this context, the aim of our research is to determine the effect of Helfer Skin Tap technique and ShotBlocker application on pain in the application of IM injection to the deltoid muscle.

\section{Research Hypotheses;}

$\mathrm{H} 1$ : Standard injection practice in IM injection is effective on injection pain.

$\mathrm{H}$ 2: The use of ShotBlocker in IM injection is effective on injection pain.

H3: Helfer Skin Tap application in IIM injection is effective on injection pain.

\section{METHODS}

Our study, which is a randomized controlled experimental study, was included 120 individuals who met then clusion criteria for IM Hepatitis B injection at Family Health Center, affiliated to Sivas Public Health Services Presidency, between 05.10.2020 and 30.12.2020. Patients were randomized by age, gender and BKI. The study was designed as a prospective, single-blind randomized controlled trial. The research was conducted in accordance with the Consolidated Standards of Reporting Trials (CONSORT) Checklist guide (Fiqure 1). When $\alpha=0.05, d=0.48$, power $(1-\beta)=0.80$ were taken to meet the parametric test assumptions, the power of the test $(p=0.821364)$ was found, and the sample was calculated as $n 1=33, n 2=33, n 3=33$. In case of loss of sample during the research, $10 \%$ more of the calculated sample was taken and 40 individuals were included in each group. While the groups were randomized, "application group-I", "application group-II and" control group-I "were written on a card. A total of 120 patients including the Helfer Skin Tap Technique $(n=40)$, the standard application ( $n=$ $40)$ and the ShotBlocker technique $(n=40)$ were included in the study to meet the parametric test assumptions.

Randomization: Individuals who applied to Toki Family Health Center during the study and met the inclusion and exclusion criteria were included in the study. In the literature, it is stated that injection pain may differ according to age, gender and BMI $(10,18)$. Therefore, in order to control the factors that may affect the homogeneity of the study, patients who met the inclusion criteria were stratified according to age, gender and $\mathrm{BMI}$ value and randomized into blocks. The number of people was determined according to the number of samples determined in each stratum of the four strata. Since we do not have an existing pool of individuals and individuals who applied to the Family Health Center were included in the sample, the individuals in each stratum were assigned to the research groups by block randomization method using a computer-generated list (www.randomizer.org). Group assignment was made by the Family Health Center nurse, who would not participate in data collection or statistical analysis, in sequential numbering, and group assignments were kept confidential in closed-opaque envelopes. It was determined whether the person would be in the groups during the block, according to which strata group characteristics the new individual who applied to the Family Health Center met the inclusion and exclusion criteria and whose consent was obtained. To confirm the homogeneity of the groups after randomization, the groups were compared according to age, gender and $\mathrm{BMI}$ using the chi-square test. Randomization was provided so that there was no statistically significant difference between the groups.

Blinding: While it was not possible for the researcher and the patients to be blinded to the intervention, the research data were coded as A, B and C, and entered into the database by someone other than the researcher, and statistical analyzes were carried out with coding by a biostatistics expert (statistician blinded).

The criteria for inclusion in the study were determined to be the following: (a) Speaking and understanding Turkish, (b) volunteering to participate in the study and obtaining a written consent, (c) aged 18-65, (d) when the patient's file was examined, sensory-motor deficit, (e) Hepatitis B vaccine to be administered, ( $f$ ) not receiving oral or parenteral analgesic treatment prior to administration, $(g)$ not having a general VAS score above 0 before injection, i.e. no general pain Patients with (h) orientation of place and time, (I) without vision and hearing problems were included in the 
study. Research Personal Information Form and Visual Analog Scala (VAS) were used to collect data of the study.

The exclusion criteria from the study were determined as follows: (a) who are not willing to participate in the research, (b)Infection, scar tissue, wound, burn, incision, etc. at the IM injection site. found patients were not included in the study.

\subsection{Personal Information Form}

This form was prepared by the researchers in line with the literature and consists of 4 items: age, gender, body mass index and fear of injection $(2,3,8,9,10)$.

\subsection{The Visual Analogue Scale (VAS)}

The scale was first used in the 1970s. It was defined by Selby et al in the 1980s to assess the quality of life in cancer patients (20). VAS has been used in many studies evaluating different parameters after the 1990s, and it has recently been used to measure special conditions such as pain. The test has proven itself for a long time, and it is widely accepted and easily applicable in the world literature. It is a $10 \mathrm{~cm}$-long scale, the left end of which is for "no pain" and the right end of which is for "severe pain", on which the individual can mark the level of their pain VAS is used to convert some non-quantifiable values into numeric values $(21,22)$.Two end definitions of the parameter to be evaluated are written on both ends of a 100 $\mathrm{mm}$ line, and the individual is asked to indicate their pain status to the appropriate point on this line by drawing a line, marking a point, or marking a sign. The length of the distance from the point showing "no pain" to the point which the individual marked indicates the individual's pain. The most important advantage of the scale is that it does not use a language and is easy to apply. Whether the alignment of the line on which the test is applied is vertical or horizontal, or its length does not affect the result of the measurement. VAS is reported to be more sensitive and reliable than other onedimensional scales for measuring pain severity $(21,22)$.

\subsection{Administration of The Data Collection Tools}

Before starting the study, approval was obtained from Clinical Research Ethics Committee and then from the center where the application was performed. In order to apply the study, the researcher was in the Family Health Center for 5 days during the week between 08.00-17.00 in accordance with the working hours of the Family Health Center. In the study, all injections were used by the same ASM nurse in order not to affect the decision of the individuals, while the approaches to reduce pain were taken by researcher carried out by (Figure 1). VAS was applied to the individuals included in the study before injection and the individual with a score above 0 , that is, experiencing a general pain, was not included in the study on the grounds that individual could not evaluate the pain due to injection correctly. Patients were randomized according to their age, gender and BKi. While the groups were randomized, "application group-l", "application group-II and" control group-I "were written on a card. A total of 120 patients including the Helfer Skin Tap Technique $(n=40)$, the standard application $(n=40)$ and the ShotBlocker technique $(n=40)$ were included in the study to meet the parametric test assumptions. The individuals in the first group were injected with Hepatitis B vaccine by the same ASM nurse using the Helfer Skin Tap Technique, and then the pain level was determined using VAS. Hepatitis B vaccine was injected to the individuals in the second group by the same ASM nurse using the standard technique in line with the injection administration protocol, and then the pain and level were determined using VAS. Individuals in the third group were injected with Hepatitis $B$ vaccine by the same ASM nurse using Shot Blocker, and then the pain level was determined using VAS (Figure 2).

The application protocols used in the research were prepared by the researchers in line with the guidelines in the literature $(4,7,8,10)$ (Figure 1).

\begin{tabular}{|l|l|}
\hline Medicine & HBVAXPRO $10 \mathrm{mcg} / 1 \mathrm{ml}$ \\
\hline Injector Volume & $5 \mathrm{ml}$ \\
\hline Needle number & 21 \\
\hline Needle point replacement & Every injection \\
\hline Air lock technique & $0.2 \mathrm{ml}$ \\
\hline Injection Area & Deltoid area \\
\hline Injection site cleaning & $70 \%$ ethyl alcohol \\
\hline $\begin{array}{l}\text { Needle entry and exit } \\
\text { angle }\end{array}$ & 90 derece \\
\hline Injection time & $1 \mathrm{ml} / 10$ sn \\
\hline After injection & $\begin{array}{l}\text { Light pressure on the injection area, no } \\
\text { massage }\end{array}$ \\
\hline $\begin{array}{l}\text { The person who recorded } \\
\text { the data }\end{array}$ & Researcher \\
\hline
\end{tabular}

Figure 1. Intramuscular Injection Protocol

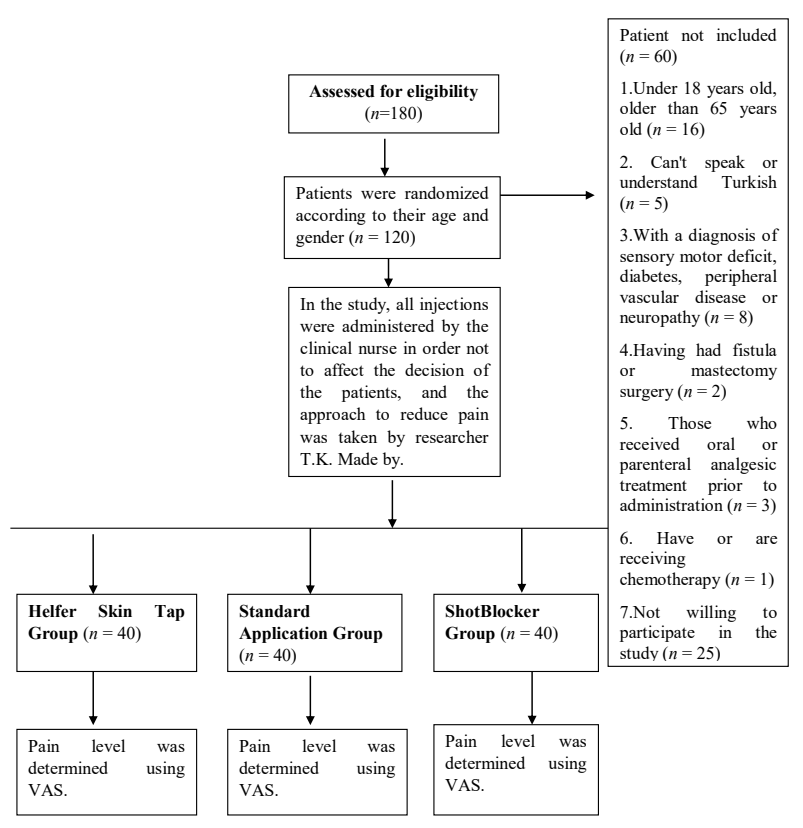

Figure 2. Flow diagram 


\subsection{Ethical Consideration}

The study was carried out in accordance with the Declaration of Helsinki 2008 Principles and at the outset, the approval of Sivas Cumhuriyet University Faculty of Medicine Clinical Research Ethics Committee (Decision No: 2020-06/05) and the institutional permission were obtained. After the individuals included in the study were informed and their informed consent was obtained, the researcher started collecting the study data. The individuals were made sure that that the decision on whether or not to participate in the study totally belonged to them, the data obtained from this study would be used only within the scope of the research, and that confidentiality would definitely be ensured.

\subsection{Evaluation of Data}

Analysis of the study data was performed using IBM SPSS Statistics Version 20.0 (IBM, Inc., Armonk, NY, USA) and tables were created.In statistical analysis; Descriptive statistics and frequency distributions of demographic data are given. Since scale is used while determining our data, nonparametric statistical methods are used. In statistical analysis, Kruskal Wallis test and Mann Whitney $U$ test were used and the level of error was taken as 0.05 .

\section{RESULTS}

In Table 1, the distribution of the individuals included in the study according to some descriptive characteristics is given. When the distribution of individuals according to their gender is examined; $60 \%$ of the Helfer skin tap group, $62.5 \%$ of the standard application group and $60 \%$ of the ShotBlocker group consists of female individuals. $90 \%$ of individuals in the helfer skin tap group, $80 \%$ in the standard application group, and $90 \%$ in the shotblocker group are between the ages of 18-34. Again, $75 \%$ of the individuals' helfer skin tap group, $72.5 \%$ of the standard application group, and $70 \%$ of the ShotBlocker group's BMI is $18.50-24.99 \mathrm{~kg} / \mathrm{m} 2$. In our study, $75 \%(n=30)$ of the Helfer Skin Tap group, $82.50 \%(n=33)$ of the standard application group, and $75 \%(n=30)$ of the Shot Blocker group reported that they had no fear of needles (Table 1 ).

When the demographic characteristics of the individuals and the distribution of the mean pain scores after IM injection were examined according to different injection methods, there was no statistically significant difference between the gender, BMI values and injection fear conditions of the individuals $(p>0.05)$, the individuals aged 41-64 were Mean pain scores were found to be statistically significantly lower than those in the $18-40$ age group $(p<0.05)$ (Table 2$)$.

When the distribution of post-injection pain score averages of individuals according to different injection methods were examined, it was calculated that the average pain score of the Helfer Skin Tap group was $4.00(2.00,6.00)$ and 7.00 $(4.00,9.00)$ in the standard application group and 3.00 (2.00, $4.00)$ in the ShotBlocker group. The difference between the average pain scores of the different methods used for pain control in IM injection application was found to be significant and statistically significant ( $p=0.001$ ) (Table 3$)$.

Table 1. Distribution of Individuals Received Intramuscular Injection by Some Descriptive Features

\begin{tabular}{|c|c|c|c|}
\hline Characteristic & $\begin{array}{c}\text { Helfer Skin Tap } \\
\text { Group } \\
n \%\end{array}$ & $\begin{array}{c}\text { Standard } \\
\text { Application Group } \\
n \%\end{array}$ & $\begin{array}{c}\text { ShotBlocker } \\
\text { Group } \\
n \%\end{array}$ \\
\hline \multicolumn{4}{|l|}{ Gender } \\
\hline Female & $24(60 \%)$ & $25(62.5 \%)$ & $24(60 \%)$ \\
\hline Male & $16(40 \%)$ & $15(37.5 \%)$ & $16(40 \%)$ \\
\hline \multicolumn{4}{|l|}{ Age } \\
\hline $18-40$ & $36(90 \%)$ & $32(80 \%)$ & $36(90 \%)$ \\
\hline 41-64 & $4(10 \%)$ & $8(20 \%)$ & $4(10 \%)$ \\
\hline \multicolumn{4}{|l|}{ BMI } \\
\hline $\begin{array}{l}18.50-24.99 \\
\mathrm{~kg} / \mathrm{m}^{2}\end{array}$ & $30(75 \%)$ & $29(72.5 \%)$ & $28(70 \%)$ \\
\hline $\begin{array}{l}25.00-29.99 \\
\mathrm{~kg} / \mathrm{m}^{2}\end{array}$ & $10(25 \%)$ & $11(27.5 \%)$ & $12(30 \%)$ \\
\hline \multicolumn{4}{|c|}{\begin{tabular}{|l|} 
Fear of needles \\
\end{tabular}} \\
\hline Evet & $10(25 \%)$ & $7(17.5 \%)$ & $10(25 \%)$ \\
\hline Hayır & $30(75 \%)$ & $33(82.5 \%)$ & $30(75 \%)$ \\
\hline
\end{tabular}

Table 2. Demographic Characteristics of Individuals and Distribution of Post-Injection Pain Scores According to Different Injection Methods

\begin{tabular}{|c|c|c|c|}
\hline Characteristic & $\begin{array}{l}\text { Helfer Skin Tap } \\
\text { Group }(n=40)\end{array}$ & $\begin{array}{l}\text { Standard } \\
\text { Application } \\
\text { Group }(n=40)\end{array}$ & $\begin{array}{l}\text { ShotBlocker } \\
\text { Group }(n=40)\end{array}$ \\
\hline Gender & \multicolumn{2}{|c|}{ Median (min-max) } & \\
\hline Female & $4.00(2.00,6.00)$ & $7.00(4.00,9.00)$ & $\begin{array}{c}3.00(2.00 \\
4.00)\end{array}$ \\
\hline Male & $3.00(2.00,6.00)$ & $6.00(4.00,9.00)$ & $\begin{array}{c}3.00(2.00 \\
4.00)\end{array}$ \\
\hline \begin{tabular}{|l|} 
Statistical \\
Result $(p)$
\end{tabular} & $p=0.315$ & $p=0.224$ & $p=0.976$ \\
\hline \multicolumn{4}{|l|}{ Age } \\
\hline $18-40$ & $4.00(2.00,6.00)$ & $7.00(4.00,9.00)$ & $\begin{array}{c}2.00(2.00 \\
2.00)\end{array}$ \\
\hline $40-64$ & $4.00(3.00,5.00)$ & $8.50(7.00,9.00)$ & $\begin{array}{c}3.00(2.00 \\
4.00)\end{array}$ \\
\hline $\begin{array}{l}\text { Statistical } \\
\text { Result }(p) \\
\end{array}$ & $p=0.690$ & $p=0.020$ & $p=0.025$ \\
\hline \multicolumn{4}{|l|}{ BMI } \\
\hline $\begin{array}{l}18.50- \\
24.99 \mathrm{~kg} / \mathrm{m}^{2}\end{array}$ & $4.00(2.00,6.00)$ & $7.00(4.00,9.00)$ & $\begin{array}{c}3.00(2.00 \\
4.00)\end{array}$ \\
\hline $\begin{array}{l}25.00-29.99 \\
\mathrm{~kg} / \mathrm{m}^{2}\end{array}$ & $4.00(3.00,5.00)$ & $7.00(4.00,9.00)$ & $\begin{array}{c}3.00(2.00 \\
4.00)\end{array}$ \\
\hline $\begin{array}{l}\text { Statistical } \\
\text { Result }(p)\end{array}$ & $p=0.863$ & $p=0.530$ & $p=0.640$ \\
\hline \multicolumn{4}{|l|}{ Fear of needles } \\
\hline Yes & $4.00(2.00,6.00)$ & $8.00(4.00,9.00)$ & $\begin{array}{c}2.00 \\
(2.00,3.00) \\
\end{array}$ \\
\hline No & $3.50(2.00,6.00)$ & $7.00(4.00,9.00)$ & $\begin{array}{c}3.00 \\
(2.00,4.00) \\
\end{array}$ \\
\hline $\begin{array}{l}\text { Statistical } \\
\text { Result }(p)\end{array}$ & $p=0.559$ & $p=0.073$ & $p=0.098$ \\
\hline
\end{tabular}


Table 3. Distribution of the Individuals Mean Pain Points PostInjection According to Different Injection Methods

\begin{tabular}{|c|c|c|c|}
\hline Pain scores & $\begin{array}{l}\text { Helfer Skin Tap } \\
\text { Group }(n=40)\end{array}$ & $\begin{array}{l}\text { Standard } \\
\text { Application } \\
\text { Group }(n=40)\end{array}$ & $\begin{array}{l}\text { ShotBlocker } \\
\text { Group }(n=40)\end{array}$ \\
\hline \multicolumn{4}{|l|}{ Median (min-max) } \\
\hline $\begin{array}{l}\text { Pain scores post } \\
\text { IM Injection }\end{array}$ & $4.00(2.00,6.00)$ & $7.00(4.00,9.00)$ & $\begin{array}{c}3.00(2.00 \\
4.00)\end{array}$ \\
\hline \multicolumn{4}{|c|}{ Statistical Result $p=0.001$} \\
\hline
\end{tabular}

\section{DISCUSSION}

Pain is a complex and multidimensional condition associated with actual or potential tissue damage, leading to unpleasant sensory and emotional experiences. It is also an individual, unique, and subjective experience that can be difficult to describe and describe (11). Prevention and relief of pain is the most fundamental requirement of human rights. Therefore, using the best approach in pain management is one of the primary responsibilities of the nurse $(23,24)$.

Nurses are responsible for relieving individuals with the methods and techniques they apply in care $(11,25)$. In the literature, it is stated that gender is important among the factors affecting pain behavior in individuals, and men can tolerate pain better with the effect of universal and social reasons $(11,26)$. However, in our study, the difference between the pain levels of men and women due to IM injection application was found to be statistically insignificant ( $p>0.05)$, and there was no difference between genders in responses to acute pain in IM injection application (Table 2).

The investigation of the causes of intramuscular injectioninduced pain demonstrated that the length and thickness of the needle the injection site the technique used the amount and the physical and chemical properties (osmolarity, $\mathrm{pH}$, concentration and auxiliary chemicals) of the drug injected were effective $(10,27)$. Within this context, all the IM injections in our study were administered in accordance with the standard injection protocol consistent with the guidelines in this field (Fiqure 1).

In our study, it was found that the average pain scores of individuals aged 41-64 in standard and Shot Blocker application were statistically significantly lower than those in the 18-40 age group (Table 2). Considering the physiological changes and regressions in the transmission and perception of pain with the advancement of age, it may be an expected result that elderly individuals experience less pain after injection. Different results were obtained in the studies conducted on this subject, and it was reported that the pain experienced after injection in older ages was higher than in other age groups $(23,28)$.

When the mean post-injection pain scores of the individuals were examined according to their BMI values, the difference between the pain levels of the individuals in all three groups due to IM injection applications was found to be statistically insignificant ( $p>0.05)$ (Table 4).
However, when the literature is examined, it has been stated that the perceived pain associated with injection is less in individuals with thick subcutaneous adipose tissue $(18,29)$. Our research does not show parallelism with the literature in this aspect.

Considered as an integral part of healthcare services, IM injections are widely used in treatment processes, and as an invasive procedure, they often cause pain $(10,16)$. Because the injection area is small among the IM injection sites, the most painful area is the deltoid area (8). In this context, it is extremely important to use pain control approaches in IM injection applied to this area $(11,18)$. Various methods and techniques are used to reduce the pain caused by injection applications and to increase the comfort of the individual (11). In our study, the mean pain scores of the Helfer Skin Tap technique, which is used to provide pain control in IM injection application, compared to the standard application, and the use of Shot Blocker was found to be statistically significantly lower than the Helfer Skin Tap and standard application. When the studies using the Helfer Skin Tap technique in the literature were examined, Jyoti et all., (2018) found that the use of the Helfer Skin Tap technique to provide pain control in IM injection application was found to be significantly effective in reducing pain due to injection (26). Again, Soliman and Hassnein (2016) found that IM injections using the Helfer Skin Tap technique were an effective method in reducing IM injection pain compared to the standard technique (27). When the use of ShotBlocker was examined in the literature, Aydın \&Avşar (2019) found that the administration ShotBlocker on reduced injection pain (23). Çağlar et al., (2017) determined that the use of ShotBlocker during hepatitis $B$ vaccine administration to newborns is effective in reducing injection pain (29). Çelik and Khorshid (2015) found that pain in IM injection using ShotBlocker was significantly less than the control and placebo groups in their study with individuals over the age of 18. (18). Our research supports the literature in this respect. In our study, when the relationship between the pain levels experienced after IM injection using different non-pharmacological methods was examined, it was determined that the pain experienced due to injection could be reduced with the use of nonpharmacological methods. In this context, the application of different methods for pain control and the evaluation of their effectiveness are very important and necessary in terms of evidence-based applications.

\section{CONCLUSION}

In line with the findings obtained in our study, it was concluded that the use of ShotBlocker was more effective than the Helfer Skin Tap and the standard application group in reducing the pain due to injection in IM injection application, and the Helfer Skin Tap technique was more successful than the standard application.

In this context, it is recommended to conduct similar studies in different populations for the Helfer Skin Tap and ShotBlocker application in order to standardize the 
applications and create evidence in IM injection application. In line with these results, it is recommended that health professionals, especially nurses, use non-pharmacological methods with proven efficacy more frequently to relieve pain in IM injection, follow the developments in this field and put them into practice

\section{REFERENCES}

[1] Ağaç E, Güneş ÜY. Effect on pain of changing the needle prior to administering medicine intramuscularly: A randomized controlled trial. Journal of Advanced Nursing 2011; 67 (3):563-568.

[2] Nicoll LH, Hesby A. Intramuscular injection: An integrative research review and guideline for evidence-based practice. Applied Nursing Research 2002;15 (3): 149-162.

[3] Legrand G, Guiguet-AuclairC, Viennet H, Aumeran C, Reynaud D, Badrikian L, Debost-Legrand A. Nurses' practices in thepreparation and administration of intramuscular injections in mental health: A cross-sectional study. Journal of ClinicalNursing2019; 28(17-18): 3310-3317.

[4] Zhuo P, Gao D, Xia Q, RanD, Xia W. Sciatic nerve injury in children after gluteal intramuscular injection: Case reports on medical malpractice. Medicine, Science and theLaw2019; 59(3), 139-142.

[5] Kara D, Yapucu G. The Effect on pain three different methods of intramuscular injection: a randomized controlled trial. International Journal of Nursing Practice 2016; 22 (2):152-159.

[6] Kara D, Dikmen Y, Kokturk F, Dedeoğlu Y. The Effect of air-lock technique on pain at the site of intramuscular injection. Saudi Medical Journal 2016; 37 (3): 304-308.

[7] Mitchell JR, Whitney FW. The Effect of injection speed on the perception of intramuscular injection pain. A clinical update. Workplace Health \&Safety Journal 2011; 49 (6): 286-292.

[8] McWilliam P.L, Botwinski CA, La Course JR. Deltoid intramuscular injections and obesity. Medical Surgical Nurses 2014; 23(1): 4-7.

[9] Kusumadevi MS, Dayananda G, Shivakumar V, Elizabeth J, Kumudavathi MS. The perception of intramuscular injection pain in men vs women. Biomedical Research 2011; 22 (1): 107-110.

[10] Cocoman A, Murray J. Intramuscular injections: A rewiev of best practice for mental health nurses. Journal of Psychatric and Mental Health Nurses 2008; 15: 424-434.

[11] Karabey T, Karagözoğlu Ş. Use of non-pharmacological methods for pain control in intramuscular injection applications: a systematic review. IOSR Journal of Nursing and Health Science 2020; 9(2):1-6.

[12] Helfer J.K. Painless Injections: Helfer skin tap technique. Nurse educator 2000; 25(6): 272-273.

[13] Dimpleshree K, Ramasambasivan R, Mahariba SK, Princy K. Effectiveness of helfer skin tap technique on pain experience among the patients receiving intramuscular injection. TNNMC Journal of Medical \& Surgical Nursing 2020; 8(1): 24-30.

[14] Khanra S, Asokan R, Lenka A. Helfer skin tap technique on pain associated with intramuscular injection among adult patients. International Journal of Nursing Education 2018; 10(3): 12-17.
[15] Sivri Bilgen B, Balcı S. The effect on pain of Buzzy ${ }^{\circledR}$ and ShotBlocker ${ }^{\circledR}$ durin gheadministration of intramuscular injections to children: A randomized controlled trial. Journal of Korean Academy of Nursing 2019; 49(4): 486-494.

[16] Bilge S, Aydin A, Gun, C, Aldinc H, Acar YA, Yaylaci S, Balci V. Comparison of the efficacy of ShotBlocker and cold spray in reducing intramuscular injection-related pain in adults. Saudi Medical Journal 2019; 40(10): 996-1002.

[17] Yilmaz G, Alemdar DK. Using Buzzy, Shotblocker, and bubble blowing in a pediatric emergency department to reducethepain and fear caused by intramuscularinjection: $A$ randomized controlled trial. Journal of Emergency Nursing 2019; 45(5): 502-511.

[18] Çelik N, Khorshid L. Theuse of ShotBlocker for reducing the pain and anxietyassociatedwithintramuscularinjection. Holistic Nursing Practice 2015; 29(5): 261-271.

[19] Tuğrul E, Çelik N, Khorshid L. Effects of ShotBlocker on relief of pain due to hepatitis $b$ vaccine injection into deltoid muscle. International Journal of Caring Sciences 2017; 10(3): 1669-1675.

[20] Selby PJ, Chapman JA, Etazadi Amoli J, Dalleyand D, Boyd NC. The development of a method for assessing the quality of life of cancer patients. British Journal of Cancer 1984; 50(3):13.

[21] Bijur PE, Silver W, Gallagher EJ. Reliability of the visual analog scale for measurement of acute pain. Academic emergency medicine 2001; 8(12): 1153-1157.

[22] Cline M.E, Herman J, ShowF, Marton RD. Standardization of the Visual Analogue Scale. Nursing Research 1992; 41 (6): 378-379.

[23] Aydin E, Avşar G. Examining the effect of Shotblocker in relieving pain associated with intramuscular injection. Complementary therapies in medicine 2019; 47; 102192.

[24] Hylands-White N, Duarte RV, Raphael JH. An overview of treatment approaches for chronic pain management. Rheumatology International 2017; 37(1): 29-42.

[25] Nakajima Y, Fujii T, Mukai K, Ishida A, Kato M, TakahashiM, Ozaki N. Anatomically safe sites for intramuscular injections: a cross-sectional study on young adults and cadavers with a focus on the thigh. Human Vaccines \& Immunotherapeutics 2020; 16(1):189-196.

[26] Jyoti G, Arora S, Sharma B. Helfer Skin Tap Technique for the IM injection pain among adult patients. Nursing and Midwifery Research,2018; 14(3): 18.

[27] NS762 Ogston-Tuck, S. Intramuscular injection technique: an evidence-based approach. Nursing Standard 2014; 29(4), 52-59.

[28] Soliman HMM, Hassnein AA. Efficacy of Helfer skin tapping technique on pain intensity as perceived by the patients receiving intramuscular injection. International journal of Nursing Didactics 2016; 6(2): 12-22.

[29] Özdemir L, Pınarcl E, Akay BN, Akyol A. Effect of Methylprednisolone injection speed on the perception of intramuscular injection pain. Pain Management Nursing 2010; 1-8.

[30] Çaglar S, Büyükyilmaz F, Cosansu G, Çaglayan S, Effectiveness of ShotBlockerfor immunization pain in full-term neonates: A randomized controlled trial. The Journal of Perinatal \& Neonatal Nursing 2017; 31 (2): 166-171. 